\title{
Erratum to: Effects of maltodextrin glycosylation following limited enzymatic hydrolysis on the functional and conformational properties of soybean protein isolate
}

Yating Zhang $\cdot$ Chen Tan $\cdot$ Xiaoming Zhang $\cdot$

Shuqin Xia - Chengsheng Jia - Karangwa Eric •

Shabbar Abbas $\cdot$ Biao Feng $\cdot$ Fang Zhong

Published online: 18 April 2014

(C) Springer-Verlag Berlin Heidelberg 2014

Erratum to: Eur Food Res Technol

DOI 10.1007/s00217-014-2164-5

In the original publication, the acknowledgement was incorrectly published.

The correct acknowledgement should read as:

"This research was financially supported by projects of the national 125 Program of China (2011BAD23B04 and 2012BAD33B05)".

The online version of the original article can be found under doi:10.1007/s00217-014-2164-5.

Y. Zhang $\cdot$ C. Tan $\cdot$ X. Zhang $(\bowtie) \cdot S$. Xia $\cdot$ C. Jia $\cdot$ K. Eric

S. Abbas $\cdot$ B. Feng $\cdot$ F. Zhong

State Key Laboratory of Food Science and Technology,

School of Food Science and Technology, Jiangnan University,

Lihu Road 1800, Wuxi 214122, Jiangsu, China

e-mail: xmzhang@jiangnan.edu.cn

Y. Zhang

e-mail: yolanda3788@163.com 